
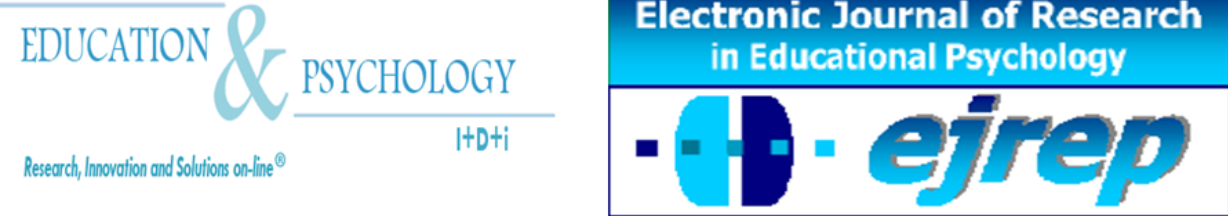

Editorial EOS

\title{
Importancia de los amigos y los padres en la salud y el rendimiento escolar
}

\section{Agustín Ernesto Martínez González ${ }^{1}$, Cándido José Inglés Saura ${ }^{2}$ José Antonio Piqueras Rodríguez ${ }^{2}$, Victoriano Ramos Linares,}

${ }^{1}$ Departamento de Investigación y Tratamiento, Clínica Neuropsicología Mayor, Alcantarilla (Murcia)

${ }^{2}$ Departamento de Psicología de la Salud, Universidad Miguel Hernández, Elche (Alicante)

${ }^{3}$ Servicios Sociales de Callosa de Segura (Alicante)

\section{España}

Correspondencia: Agustín Ernesto Martínez González. C/ Mayor, $1134^{\circ}$ C. 30820 Alcantarilla (Murcia). España. E-mail: cpcmayor@yahoo.es 


\section{Resumen}

En este artículo detallamos los estudios que hablan del papel clave de los amigos y los padres en el bienestar emocional, físico, así como en el rendimiento académico y laboral a lo largo de la vida. Igualmente, hablamos de los estilos educativos de los padres y su influencia en el desarrollo del estado emocional, relación social y rendimiento académico. Destacamos la importancia de desarrollar una conducta prososocial en los jóvenes para desarrollar mejores niveles de estabilidad emocional y rendimiento académico.

Palabras Clave: estilo educativo, conducta prosocial, cooperación, salud, bienestar, salud mental.

Recibido: 22/06/09 Aceptación Inicial: 25/06/09 Aceptación Definitiva: 25/11/09 


\title{
The importance of friends and parents in health and academic performance
}

\begin{abstract}
In this article we detail the studies that talk about the key role of friends and parents in the emotional, physical and academic performance and work throughout their lives. Also, we discuss the educational style of parents and their influence on the development of emotional, social and academic performance. We stress the importance of developing a prososocial behavior in young people to develop higher levels of emotional stability and academic achievement.
\end{abstract}

Keywords: educational style, prosocial behavior, cooperation, health, wellness, mental health.

Received: 06/22/09 Initial Acceptance: 06/25/09 Definitive Acceptance: 11/25/09 


\section{Introducción: Evolución de las relaciones sociales}

En el siguiente artículo trataremos de exponer los aspectos más importantes del desarrollo de las relaciones sociales en el ser humano a lo largo de su vida. Destacaremos el papel que juegan las relaciones sociales tanto en el bienestar emocional y físico como en el rendimiento académico y laboral en la juventud y edad adulta. Se discute el papel del estilo educativo en el desarrollo de estilos de conducta y sus implicaciones en el bienestar emocional, el rendimiento académico y laboral.

A lo largo de la vida van desarrollándose tanto la personalidad como los procesos cognitivos y psíquicos del ser humano. En este desarrollo juegan un papel muy importante las relaciones sociales (Inglés, 2009). Así, dichas relaciones van variando dependiendo del periodo evolutivo en el que se encuentra el individuo. Durante la niñez, la referencia y apoyo principal recae en la figura paterna y materna, ya que el niño precisa de una orientación constante debido a la inmadurez cognitiva. En el período de la adolescencia, aparecen una serie de cambios importantes tanto en el desarrollo físico, mental, emocional, com en las relaciones interpersonales. Así, en este periodo, el individuo tiene una serie de ambivalencias y contradicciones ya que se encuentra en un proceso de búsqueda del equilibrio consigo mismo y con la sociedad (Inglés, 2009; Siverio y García, 2007; Smetana, 1989).

La evidencia empírica previa ha puesto de manifiesto que durante la adolescencia aparecen cambios en las relaciones familiares (Inglés, 2009). Los adolescentes pasan más tiempo fuera de casa y disminuye el número de interacciones positivas con los padres, apareciendo conflictos relacionados con aspectos de la vida cotidiana tales como las tareas de casa, las amistades, la forma de vestir o la hora de volver a casa (Galambos y Almeida, 1992; Smetana, Braeges y Yau, 1991). De esta forma, las relaciones con los compañeros van ganando importancia, intensidad y estabilidad, y el grupo de iguales se convierte en el contexto de socialización más influyente (Inglés, 2009; Lynch y Cicchetti, 1997; Mayseless, Wiseman y Hai, 1998; Rice, 1997; Sletta, Valas y Skaalvik, 1996).

Como consecuencia de la maduración cognitiva y del tiempo que dedican a hablar de sí mismos, los adolescentes se comprenden mejor así mismos y a los otros, lo que va a repercutir en que las relaciones con los amigos estén marcadas por la reciprocidad y el apoyo mu- 
tuo (Volling, Youngblade y Blesky, 1997). Además, aumentará sustancialmente la intimidad de estas relaciones, sobre todo entre las chicas durante la adolescencia temprana y media (Bracken y Crain, 1994). De esta manera, se produce una expansión de las redes extrafamiliares, de modo que los jóvenes se ven expuestos a un amplio abanico de nuevas situaciones sociales (fiestas, bares, oficinas públicas, establecimientos comerciales, etc.), donde se relacionan con personas desconocidas o no allegadas (Flores y Díaz, 1995), apareciendo una intensificación de las relaciones con compañeros del mismo sexo (Buhrmester y Furman, 1992; Mayseless et a., 1998) y del inicio de las relaciones románticas con el otro sexo (Bracken y Crain, 1994; Furman y Buhrmester, 1992). Por otro lado, los jóvenes van buscando nuevas experiencias para conseguir la aceptación y respeto de los iguales $\mathrm{y}$, al mismo tiempo, comienzan a establecer un margen cada vez más amplio en la autonomía y en las relaciones con los padres (Jessor, 1988). Por lo tanto, en la adolescencia la necesidad de afiliación al grupo de iguales es un hecho común y necesario para el desarrollo evolutivo. Sin embargo, el motivo de afiliación hacia el grupo de iguales varía a lo largo del ciclo evolutivo. Así, durante la preadolescencia, los jóvenes suelen tener dificultades a la hora de mostrar desacuerdo ante la presión del grupo de iguales y se muestran insatisfechos en relación con los iguales, ya que desean tener más amigos/as, mientras que en la adolescencia media comienzan a sentirse integrados en el grupo de iguales, aunque dicha afiliación no suponga un aporte de apoyo o de comunicación (Rodrigo et al., 2004).

En esta línea, debemos considerar que resulta erróneo pensar que los vínculos con el grupo de iguales suponen una disminución de la influencia de los padres durante la juventud (Laursen, Coy y Collins 1998; Rice, 1997). Al contrario, un gran número de investigaciones que estudian los conflictos familiares durante la adolescencia, hallan que a partir de la pubertad hay una clara correlación negativa entre edad y número de conflictos entre padres e hijos. Así, conforme los jóvenes afirman su individualidad y autonomía, no se distancian de sus padres, sino que requieren, con mayor intensidad, el apoyo o guía de éstos, produciéndose un acercamiento entre ellos (Mayseless, Wiseman y Hai, 1998; Rice y Mulkeen, 1995), debido, quizás, a que conforme los chicos y chicas se acercan a la adultez su capacidad para ponerse en lugar de sus padres se ve incrementada (Smetana, 1989).

Así pues, concluimos que tanto los padres como los amigos no compiten entre sí, sino que representan influencias complementarias que satisfacen diferentes necesidades de los jóvenes (Clark-Lempers, Lempers y Ho, 1991; Lempers y Clark-Lempers, 1992). Además, las 
influencias sobre su conducta variarán según el asunto que les preocupe, por lo que es más probable que los jóvenes escuchen a los padres cuando se trata de cuestiones morales, educativas, o que tengan que ver con el dinero o con el control de relaciones interpersonales distintas de las que tienen con los compañeros. Por el contrario, los jovenes son más susceptibles de escuchar a sus compañeros cuando se trata de elegir a amigos, controlar las relaciones con los compañeros o pasar el tiempo libre (Inglés, 2009; Smetana, 1993).

Durante la edad adulta las relaciones sociales van estabilizándose y la capacidad de empatía o capacidad para ponerse en lugar de otros está más desarrollada (Eisenberg, 1990; Eisenberg, Carlo, Murphy y Van Court, 1995; Eisenberg, Miller, Shell, McNalley y Shea, 1991; Eisenberg, Zhou y Koller, 2001) debido, en parte, a la madurez cognitiva que propicia el desarrollo de la empatía, del razonamiento moral prosocial y de la capacidad de toma de perspectiva respecto a los estados internos y externos de los demás. Según la teoría motivacional de Huberman (1974), en la edad adulta el interés responde principalmente a la vida profesional, vida familiar, asuntos sociales, intereses creativos, salud física y mental. Así a lo largo de los años el adulto va variando sus necesidades y relaciones sociales; (a) en el periodo comprendido entre los 18- 30 años el adulto posee una identidad psicológica consolidada y estable. En este sentido, en la adultez, el individuo intenta conseguir su identidad social, esta vez, a través del trabajo, la carrera u oficio, para lograr un reconocimiento social y un status. Igualmente, en este periodo la persona adulta persigue una ideología propia, elige compañeros/as para su vida y quiere presentar una buena imagen de sí mismo a los demás; (b) posteriormente, entre los 30-40 años, el adulto comienza a tener un aumento en la experiencia y competencia dentro del campo profesional. Este es un periodo de estabilidad más evidente en el que el adulto se encuentra en el nivel máximo de sus potencialidades, fuerza y energía. Del mismo modo, los intereses o motivaciones que le mueven a seguir viviendo son la crianza y educación de los hijos, la competencia en el mundo profesional y laboral. El adulto suele iniciar el interés por actividades y responsabilidades comunes al grupo, así como por asuntos económicos; (c) entre a los 40-50 años, el adulto se encuentra en la cima o cumbre del ciclo vital. Toda su energía, su actividad vital está orientada hacia el mundo. Los asuntos sociales y públicos le atraen y le motivan, alcanzando los temas políticos. Además de que este periodo el adulto encuentra recompensa con percibir el crecimiento de sus hijos, a los que ve desarrollarse; (d) a partir de los 50-60 años aparece una disminución de las potencialidades físicas del adulto. En esta etapa el adulto se esfuerza por no perder terreno en su profesión o carrera. El mundo es percibido de forma más complicada que en periodos anteriores. Se hace más realis- 
ta, las motivaciones que le mueven vienen dadas por las recompensas a corto plazo que las de largo plazo. Sus intereses académicos son cada vez más filosóficos y religiosos; (e) entre los 60-70 años, el adulto declina más su aspecto físico, salud e incluso sus relaciones sociales. Llegan a sentir el deseo de alejarse de las relaciones y compromisos de carácter afectivo; lo que conlleva realizar actividades de carácter inmediato (entretenimiento, juegos, etc.) y contemplativo (turismo, arte, política, etc.); (f) finalmente, de los 70 años en adelante, el adulto se separa definitivamente del mundo laboral, debido a la jubilación, y por ello, disminuyen aun más las relaciones sociales. Los intereses que se mueven a esta edad son las pensiones, la salud y la dependencia con los demás. Según Erikson (1985), esta es la etapa de la integridad versus desesperación que viene determinada por el final de la vida, que para algunos adultos supone la aparición de angustia y miedo a la muerte.

\section{Influencia de los amigos y los padres en el bienestar psíquico}

Los beneficios derivados de disponer de amigos son abundantes. Las relaciones con los iguales constituyen una experiencia muy gratificante para los jóvenes, siendo un factor relevante para su socialización (Rice, 1997). En general, tener amigos es un buen indicador de buenas habilidades interpersonales y un signo de un buen ajuste psicológico posterior (Inglés, Delgado, García-Fernández y Díaz-Herrero, en prensa; Kimmel y Weiner, 1998; Kupersmidt, Coie y Dodge, 1990).

Numerosas investigaciones han hallado que el éxito en las relaciones interpersonales se encuentra relacionado positivamente con aspectos generales del funcionamiento psicosocial. Así, las relaciones interpersonales pueden influir positivamente en la mejora de la autoestima (Bijstra, Bosma y Jackson, 1994; Bijstra, Jackson y Bosma, 1995; Gilman y Huebner, 2006; Huebner, Suldo y Gilman, 2006) proporcionando bienestar (Bijstra et al., 1994; 1995) y felicidad (Argyle y Lo, 1990). Del mismo modo, el éxito en las interacciones con los demás esta relacionado positivamente con el afrontamiento de situaciones sociales conflictivas (Bijstra et al., 1994, 1995).

Igualmente, los padres también aportan un apoyo social vital en los eventos estresantes a los que se tiene que enfrentarse la juventud (Supplee, Shaw, Hailstones y Hartman, 2004; Wills, Resko, Ainette y Mendoza, 2004). Diversos estudios han destacado el papel importantísimo de la educación de los padres en la autorregulación emocional y el autoconcepto de 
los jóvenes (González-Pienda, Núñez, Álvarez y González-Pumariega, 2002; GonzálezPienda., et al, 2003; Supple, Shaw, Hailstones y Hartman, 2004). Así, se ha demostrado una relación positiva entre el apoyo familiar de los padres, los profesores y los iguales con una autoestima positiva de los jóvenes. Por lo tanto, podemos indicar que tanto los padres como los iguales actúan como factores protectores del funcionamiento desadaptativo, la depresión y la tensión o estrés social (Gilman y Huebner, 2006; Huebner et al., 2006; Inglés et al., en prensa), disminuyendo el riesgo de suicidio y trastornos psicopatológicos. Este sentido, LinShan (2001) encontró que el control psicológico esta positivamente determinado por la depresión y los conflicto padres-hijos.

El modo de comportarse de los jóvenes parece influir en las relaciones sociales entre los iguales y los padres, y viceversa, estas relaciones sociales pueden influir en el desarrollo de ciertas conductas en la juventud. Así, se ha puesto de manifiesto que ciertos individuos pueden tener dificultades en las relaciones interpersonales cuando presentan un déficit asertivo y ansiedad social o, por el contrario, cuando presentan conductas de agresividad o antisociales, llegando a tener una gran variedad de problemas psicológicos y conductuales.

Las conductas de tipo antisocial por parte de los jóvenes pueden haber sido aprendidas por modelado tanto por el grupo de iguales como por parte de los familiares, generando con ello conductas de maltrato entre iguales, abuso de poder y violencia (Del Barrio, Martín, Almeida y Barrios, 2003). Este tipo de conductas se relacionan positivamente con una variedad de problemas personales tales como la elevada hiperactividad, irritabilidad, ansiedad e inestabilidad emocional, resentimiento y suspicacia (Garaigordobil, 2005), autoconcepto negativo (Calvo, González y Martorell, 2001; Garaigordobil, 2005) y déficit en habilidades sociales (Calvo et al.,2001; Garaigordobil, 2005; Inglés, Hidalgo, Méndez e Inderbitzen, 2003; Inglés, Méndez, Hidalgo y Spence, 2003; Méndez, Inglés e Hidalgo, 2002). Estos problemas de conducta acarrean un rechazo social por parte de los adultos e incluso por parte de los iguales (Inglés et al., en prensa; Trianes et al., 2002; Wills y Resko, 2004; Wills, Resko, Ainette y Mendoza, 2004) incrementando el fracaso en las citas con el sexo opuesto (La Greca y López, 1998).

Del mismo modo, los individuos que presentan ansiedad social y evitan el contacto social con sus iguales tienen importantes consecuencias negativas tanto en el número de relaciones sociales como en el estado mental. Un estilo pasivo o sumiso supone realizar conduc- 
tas de evitación hacia las relaciones sociales con los compañeros, lo que genera sentimientos de soledad (Walters e Inderbitzen, 1998) y una actitud de rechazo e incluso de maltrato o abuso por parte de los iguales (Storch, Masia-Warner, Crisp y Klein, 2005), lo que, a su vez, repercute negativamente en el aprendizaje y en la mejora de habilidades sociales (Inglés et al., 2003; Inglés, Méndez, Hidalgo y Spence, 2003). Como consecuencia, los adolescentes pasivos y con menos habilidades interpersonales tienen más probabilidad de ser rechazados o ignorados por sus iguales (Chen, 2006; Inderbitzen, Walters y Bukowski, 1997; Inglés et al., en prensa; La Greca y López, 1998).

A diferencia de estos dos estilos de conducta que están más relacionados con trastornos psicológicos como la ansiedad social y el trastorno antisocial o negativo-desafiante, los adolescentes más asertivos y prosociales suelen tener un buen desarrollo de las amistades, tienen éxito en las citas con el sexo opuesto (Clark et al., 1994; La Greca y López, 1998), son aceptados por los demás como populares (Inglés, Hidalgo y Méndez, 2005; Inglés, Ruiz, et al., 2005; Inglés et al., en prensa), mantienen una buena interacción con sus padres y son aceptados por éstos, desarrollan mayores niveles de conductas positivas sociales, de forma que el grupo de iguales se convierte para estos en fuente de aprendizaje de conductas prosociales (Wentzel, Barry y Caldwell, 2004). Igualmente, tienen una buena autoestima y autoconcepto (Calvo et al., 2001; Garaigordobil, Cruz y Pérez, 2003; Martínez, 2009; Smith, Walker, Fields, Brookins y Seay, 1999), y se autorregulan mejor, siendo más estables emocionalmente (Martínez, 2009; Tur, Mestre y del Barrio, 2004).

En el proceso del formación de la personalidad y el autocontrol del joven, juega un papel fundamental la influencia de los padres y sus habilidades educativas (ver Tabla 1). El estilo educativo de los padres influye de forma decisiva en el desarrollo de la personalidad del individuo. El estilo educativo parental permisivo puede generar un adolescente dependiente y lábil emocionalmente. Este dato tiene una relación muy estrecha con la baja tolerancia a la frustración, la agresividad y la desobediencia cuando el adolescente no consigue lo que quiere, llegando al extremo de incumplir las normas sociales. Del mismo modo, si el estilo educativo de los padres es autoritario, puede influir en la aparición de conductas agresivas, es decir, el adolescente que se enfrenta a ciertos problemas de la vida puede reproducir estrategias de resolución de problemas basadas en la impulsividad y la agresividad como método modelado por el estilo educativo familiar. De igual modo, el estilo educativo autoritario puede crear en el adolescente sentimientos de inferioridad y trastornos de personalidad. En este sentido, se ha 
demostrado que la falta de accesibilidad y de supervisión de los padres, acompañada de la escasa o nula comunicación paterno-filial se relaciona con la tendencia de los hijos a relacionarse con compañeros conflictivos y a fomentar comportamientos de riesgo de carácter antisocial (Rodrigo, et al., 2004; Tur, Mestre y del Barrio, 2004).

Tabla 1. Representación de los estilos educativos

\begin{tabular}{lcccc}
\hline & PERMISIVO & DEMOCRÁTICO & AUTORITARIO & NEGLIGENTE \\
\hline Disciplina & Blanda & Firme & Dura & Blanda \\
\hline Afecto & Sobre-protección & Cariño & Hostilidad & Hostilidad \\
\hline Comunicación & Excesiva & Participativa & Pobre & Indiferencia \\
\hline
\end{tabular}

Una de las características del estilo educativo democrático y también del permisivo es que los padres estén receptivos a la comunicación con los hijos. Los padres receptivos tienden a aceptar y anteponer las necesidades de sus hijos a las propias y los padres no receptivos suelen rechazar las necesidades de sus hijos para anteponer las suyas (Hoffman, Paris y Hall, 1996). Sin embargo, los padres que tienen un estilo autoritario no son receptivos sino exigentes; para ellos la obediencia es una virtud que los hijos deben desarrollar, utilizan el poder de la fuerza cuando consideran que una conducta no es adecuada, por lo que esperan que se acepten las reglas sin cuestionamiento. Por otro lado, los padres permisivos que son receptivos y no exigentes, consideran que los jóvenes tienen que regular sus propias actividades y conductas, por lo que fuerzan a los hijos a obedecer las reglas. Respecto a los padres democráticos, éstos se caracterizan por ser receptivos y exigentes, ponen límites a sus hijos de manera racional, haciéndoles entender la utilidad de un cierto control y las consecuencias de la conducta. Este estilo fomenta el intercambio verbal y utiliza como disciplina básicamente la inducción y algún uso de la fuerza. La meta es conseguir que el joven acepte las normas de los demás sin perder su independencia. Finalmente, están los padres que tienen un estilo educativo negligente; no son exigentes ni receptivos, tratan de pasar el menor tiempo posible con sus hijos y realizar el mínimo esfuerzo por ellos. Estos padres son indiferentes en cuanto a las prácticas educativas y su estilo de disciplina es el poder de la fuerza (véase Tabla 1).

A la hora de realizar un análisis de constructos tales como, conducta prosocial, asertividad, aprendizaje cooperativo en el ámbito escolar y estilo educativo democrático, encontra- 
mos una serie de criterios comunes (véase Tabla 2). Todos estos constructos se han relacionado desde diferentes ámbitos de estudio; desde la Psicología Social, estudiando las conductas prosociales; desde la Psicología Educativa, estudiando los estilos educativos y el aprendizaje cooperativo; y, desde la Psicología Clínica, investigando los déficit asertivos y sus implicaciones en la salud mental.

Tabla 2. Relación conceptual entre conducta prosocial, aprendizaje cooperativo, asertividad y estilo educativo democrático

\begin{tabular}{|c|c|c|c|}
\hline Conducta Prosocial & $\begin{array}{l}\text { Aprendizaje } \\
\text { Cooperativo }\end{array}$ & Asertividad & Estilo Democrático \\
\hline $\begin{array}{l}\text { - Empatía } \\
\text { - Ayuda a los demás. } \\
\text { - Cooperación } \\
\text { - Liderazgo: Es apre- } \\
\text { ciado y bien visto en el } \\
\text { grupo } \\
\text { - Moralidad: Sabe qué } \\
\text { esta bien y qué mal. } \\
\text { - Respeta a todos por } \\
\text { igual independientemen- } \\
\text { te de su condición. } \\
\text { - Piensa que todos tie- } \\
\text { nen derechos a expresar } \\
\text { sus ideas. } \\
\text { - Intentan resolver con- } \\
\text { flictos de forma cons- } \\
\text { tructiva. } \\
\text { - Consideran que todos } \\
\text { tienen las mismas opor- } \\
\text { tunidades. } \\
\text { - Tiene claras sus metas } \\
\text { académicas y sociales. } \\
\text { - Tienen buen autocon- } \\
\text { trol emocional, autocon- } \\
\text { cepto y rendimiento } \\
\text { académico }\end{array}$ & $\begin{array}{l}\text { - Se trabaja con otros } \\
\text { para conseguir un obje- } \\
\text { tivo mutuo. } \\
\text { - Cada miembro es res- } \\
\text { ponsable de participar } \\
\text { en el grupo y realizar su } \\
\text { parte de trabajo. } \\
\text { - Todos los miembros } \\
\text { son iguales independien- } \\
\text { temente del género, raza } \\
\text { o religión. } \\
\text { - Todos tienen derechos } \\
\text { a expresar sus ideas. } \\
\text { - Se espera que todos } \\
\text { tengan la oportunidad de } \\
\text { liderar el grupo, tomar } \\
\text { decisiones eficaces y } \\
\text { resolver conflictos de } \\
\text { forma constructiva. } \\
\text { - Colaboran en el bien- } \\
\text { estar del grupo }\end{array}$ & $\begin{array}{l}\text { - Empatía } \\
\text { - Presta ayuda los } \\
\text { demás. } \\
\text { - Respeta a todos por } \\
\text { igual independientemen- } \\
\text { te de su condición. } \\
\text { - Piensa que todos tie- } \\
\text { nen derechos a expresar } \\
\text { sus ideas. } \\
\text { - Intentan resolver con- } \\
\text { flictos de forma cons- } \\
\text { tructiva. } \\
\text { - Consideran que todos } \\
\text { tienen las mismas opor- } \\
\text { tunidades. } \\
\text { - Se comunica, sabe } \\
\text { hacer críticas honestas, } \\
\text { decir que no y escuchar } \\
\text { de forma activa sin en- } \\
\text { juiciar. }\end{array}$ & $\begin{array}{l}\text { - Los padres fomentan el } \\
\text { aprendizaje cooperativo y } \\
\text { participan activamente. } \\
\text { - Los padres fomenta la } \\
\text { responsabilidad en el hijo. } \\
\text { - Trata a todos los hijos } \\
\text { por igual, sin favoritismos } \\
\text { ni comparaciones. } \\
\text { - Todos tienen derechos a } \\
\text { expresar sus ideas. } \\
\text { - Se intentan resolver con- } \\
\text { flictos de forma construc- } \\
\text { tiva. } \\
\text { - Los padres colaboran en } \\
\text { el bienestar de la familia. } \\
\text { - Los padres expresan } \\
\text { firmeza en el cumplimien- } \\
\text { to de las normas. } \\
\text { - Los padres se comuni- } \\
\text { can y escuchan las necesi- } \\
\text { dades o problemas de sus } \\
\text { hijos, sin enjuiciar. }\end{array}$ \\
\hline
\end{tabular}

Consideramos que existe una relación muy estrecha entre el estilo educativo democrático y la conducta prosocial de los jóvenes. Así, la evaluación positiva que los padres hacen a sus hijos, el apoyo emocional junto con la coherencia en la aplicación de las normas, es el 
estilo de crianza más relacionado positivamente con la empatía y con el comportamiento prosocial (Markiewicz, Doyle y Brendgen, 2001; Mestre, Tur, Samper, Nácher, Cortés, 2007). Los padres democráticos parecen fomentar en sus hijos el desarrollo de la autoestima y las habilidades sociales. El control del comportamiento de los jóvenes ayuda a moldear sus impulsos y garantiza la autonomía psicológica que contribuye al desarrollo de la responsabilidad y la competencia (Hoffman, Paris y Hall, 1996). Por otra parte, ese feedback social positivo, fomenta que los jóvenes se perciban aceptados por los demás y tiendan a definir estrategias alternativas de resolución de problemas en situaciones sociales, antes de manifestar comportamientos negativos o de descontrol emocional (Katainen, Räikkönen y Keltikangas-Järvinen, 1999).

Durante la edad adulta el individuo va consiguiendo una mayor estabilidad emocional y psíquica. El adulto presenta una mayor capacidad para ponerse en lugar de los demás, su conducta es más prosocial y empática debido a la madurez cognitiva. Sin embargo, conforme aumenta la edad se aprecia una disminución de las relaciones sociales en cuanto a las relaciones con los amigos y el adulto comienza a centrarse en las relaciones de pareja y en la formación de un núcleo familiar. El adulto tiene la necesidad de formar una familia, comienza a tener una mayor estabilidad laboral y afectiva, mientras que las relaciones laborales se centran en el rendimiento laboral para conseguir el aprecio y el reconocimiento social.

Sin embargo, la mayoría de los estudios epidemiológicos sobre trastornos psicológicos destacan la aparición de síntomas psicopatológicos a principios de la edad adulta. Mas concretamente, los trastornos relacionados con la ansiedad suelen aparecer en la adolescencia (15 años), mientras que los trastornos del estado de ánimo suelen aparecer a los 26 años y los relacionados con el uso de sustancias a los 21 años (Bull World Health Organ, 2000).

\section{Influencia de los amigos y los padres en la salud física}

Una adecuada salud física va unida a un estilo de vida saludable, caracterizado por el cuidado de uno mismo respecto a la prevención de enfermedades. Para ello, el individuo tiende a realizar conductas que fomentan la salud, tales como la actividad física o deportiva que previenen la aparición de enfermedades, evitando las conductas de riesgo para la salud (consumo de drogas, relaciones sexuales imprudentes, etc.) y cuidando la dieta. 
A lo largo de los años el estilo de vida va variando. Así, durante la juventud se van adquiriendo estilos de vida más insaludables. En esta línea, Rodrigo et al. (2004), hallaron que los preadolescentes (13 años) todavía no habían mantenido relaciones sexuales, ni se habían iniciado en el consumo del tabaco, alcohol, etc., mientras que en la adolescencia media (15 años-17 años) los adolescentes comenzaban a tener estilos de vida menos saludables ya que consumían tabaco y alcohol los fines de semana.

Los iguales pueden influir de forma determinante en la aparición de conductas que fomenten la salud o la perjudiquen en el sentido de que el grupo de iguales puede mediar en la toma de decisiones a la hora de realizar actividades deportivas o, por el contrario, puede influir en la aparición de conductas de consumo de alcohol, tabaco, marihuana, etc. Del mismo modo, la forma de hacer frente a la posibilidad de decir no ante esas situaciones de consumo va a depender del estilo de conducta interpersonal del joven (pasivo, asertivo y agresivo).

Así, existe un consenso entre los investigadores que destacan la relación estrecha entre estilo de conducta agresivo y con dificultades en las relaciones interpersonales y el consumo de drogas como el alcohol y el tabaquismo en la juventud. Numerosas investigaciones han encontrado que este tipo de comportamientos desadaptativos son predictores muy potentes del consumo regular y abusivo de tabaco y alcohol (Bergen, Martin, Roeger y Allison, 2005; Blum e Ireland, 2004; Clark, 1993; De Micheli y Formigoni, 2004; Griffin, Botvin, Epstein, Doyle y Diaz, 2000; Harrier, Lambert y Ramos, 2001; Kollins, McClernon y Fuemmeler, 2005; Inglés et al., 2007; Paschall, Flewelling y Rusell, 2004; Upadhyaya, Brady, Wharton y Liao, 2003; Wu, Schlenger y Galvin, 2003).

Del mismo modo, el estrés social también puede incrementar el riesgo de consumo de drogas en los jóvenes más pasivos socialmente (Zimmermann et al., 2003) y dicha conducta pasiva junto al estrés puede generar problemas en el comportamiento alimentario (Bulik, Beidel, Duchmann, Weltzin y Kaye, 1991; Fernández et al., 2003) que desembocan en problemas físicos importantes (anemia, úlceras de estómago, etc.), incluso pudiendo llegar a provocar la muerte.

Los jóvenes con ansiedad ante las relaciones sociales (fobia social) también suelen tener sus primeros temores sociales a una edad temprana que coincide con el inicio del consu- 
mo de tabaco (Sonntag, Wittchen, Höfler, Kessler y Stein, 2000) y alcohol (Zimmermann et al., 2003). Así, diversos estudios han hallado un elevado número de sujetos con fobia social que presentan un consumo abusivo de tabaco (O'Callaghan y Doyle, 2001; Sonntag et al., 2000; Wittchen, Stein y Kessler, 1999) y alcohol (Rabe-Jablonska, Dietrich-Muszalska y Gmitrowicz, 2004; Zimmermann et al., 2003). Los jóvenes diagnosticados como fóbicos sociales describen el consumo de estas sustancias como una conducta de escape que compensa su elevado grado de ansiedad en situaciones interpersonales (Sonntag et al., 2000). Así pues, la evidencia empírica ha demostrado que la fobia social es un predictor significativo del consumo de tabaco (Sonntag et al., 2000; Wittchen, Stein y Kessler, 1999) y de alcohol (Weiller, Bisserbe, Boyer, Lepine y Lecrubier, 1996; Zimmermann et al., 2003).

Por otra parte, debemos destacar que las relaciones con los iguales también pueden influir forma positiva en la aparición de conductas prosociales (Inglés et al., en prensa; Wentzel, Barry y Caldwell, 2004). Así, los adolescentes prosociales tienen una mayor facilidad para la adquisición de hábitos y estilos de vida saludable (Rodrigo et al., 2004), por lo que tienen menos riesgo de consumir drogas. Así, diversas investigaciones han puesto de manifiesto que los jóvenes prosociales, asertivos y socialmente habilidosos, en comparación con los jóvenes antisociales, son menos proclives a manifestar conductas de riesgo para la salud, tales como el consumo de drogas legales e ilegales (Inglés et al., 2007; La Greca, Prinstein y Fetter, 2001; Martínez-González, Robles y Trujillo, 2003; Sussman, Unger y Dent, 2004).

Respecto a la implicación de los estilos educativos que ejercen los padres en las conductas de salud de los jóvenes, los investigadores coinciden en destacar que los jóvenes que proceden de hogares democráticos tienen una menor tendencia a consumir drogas. Por otra parte, también se puede decir que parecen favorecer la individualización. Los jóvenes que provienen de familias con un estilo de crianza permisivo también están individualizados, pero tienen mayor tendencia a caer en el consumo de drogas. Los jóvenes de familias autoritarias están menos individualizados y más inclinados a tener trastornos emocionales. Finalmente, los padres que rechazan o son negligentes tienen hijos más proclives a tener problemas emocionales y de conducta. Además, éstos son los que consumen mayor cantidad de drogas (Hoffmann, Paris y Hall, 1996).

A lo largo de la edad adulta la salud física va deteriorándose y las complicaciones en el organismo comienzan a aparecer. En este sentido, el apoyo social para mantener un estilo 
de vida saludable es vital. La influencia de los amigos y los familiares es importante para mantener una dieta equilibrada y evitar conductas de riesgo para la salud como es el consumo de sustancias.

Debemos de tener en cuenta que, en la edad adulta, los riesgos de padecer enfermedades cardiovasculares son más probables debido a factores tales como: un aumento del sedentarismo debido al aumento de las obligaciones conyugales y la utilización de actividades sedentarias como Internet; mala alimentación, aparición de colesterol y diabetes. Se estima que las personas sedentarias tienen aproximadamente el doble de riesgo de presentar una cardiopatía isquémica, o de morir por esta enfermedad, que las personas que son activas (Bijnen, Caspersen y Mosterd, 1994; Lee, Folsom y Blair, 2003). Sin embargo, el estilo de conducta también influye en la edad adulta. Así la personas que tienen una personalidad tipo A, es decir, personas que se caracterizan por la irritabilidad, la impulsividad y el perfeccionismo, tienen una mayor predisposición a desarrollar enfermedades cardiovasculares, HTA, angina de pecho e infarto de miocardio y accidente cerebro vascular (Álvarez et al., 2006; Schroeder, et al., 2000).

\section{El papel de los amigos y los padres en el rendimiento académico y laboral}

Los primeros estudios sobre relación social entre jóvenes y rendimiento escolar, hallaron una conexión entre amistad y el funcionamiento escolar tanto para la escuela elemental (Ladd, 1990) como para preescolar y en bebes (Vandell y Mueller, 1980). En este sentido, Ladd (1990) llegó a la conclusión de que los iguales son la "cola" que solidifica inicialmente la participación en la iniciativa escolar y que la amistad de los chicos puede ser diferente en función de lo alentadora o conflictiva que sea la relación entre ellos. Este mismo autor sugiere que la amistad y la aceptación al grupo de iguales actúan, en general, como un soporte para los chicos en el ambiente escolar y puede ayudar a la adaptación en la escuela (Ladd y Kochenderfer, 1996). Así, parece ser que los niños tienden a asociarse con aquellos que muestran su mismo nivel de motivación académica (Chen, Chang y He, 2003; Estell, Farmer, Cairns y Cairos, 2002).

Por lo tanto, el grupo de iguales va a influir de forma decisiva en la toma de decisiones en la juventud, al igual que en el estilo de conducta del adolescente (Inglés et al., en prensa). Así, el rendimiento académico también va a depender del tipo de conducta prosocial o no del 
individuo y al mismo tiempo los iguales también pueden influir en la adquisición de metas y en la elaboración de conductas prosociales orientadas al rendimiento (Adeyemi, 2008; Battistich, Schaps y Wilson, 2004; Brody et al., 2006; Dana, Scott y Sunita ,2006; Inglés et al., en prensa; Tennant, 2005).

En este sentido, la mayoría de los estudios parecen indicar que existe una relación entre conducta prosocial y éxito académico, y destacan el papel importante de la conducta prosocial como protectora del fracaso académico y predictora del éxito académico en los jóvenes (Boliang, Yan y Lei, 2005; Chen et al., 2002; Estell, et al., 2002; Inglés et al., 2009; Martínez, 2009; Redondo, 2007; Velásquez, Martínez y Cumsille, 2004).

Igualmente, los iguales también pueden influir de forma negativa en el rendimiento escolar de los jóvenes. Según diversos estudios, los adolescentes que actúan de manera agresiva y violan las normas sociales tienden a tener un rendimiento académico menor (Buhs, Ladd y Herald, 2006; Eisenberg, Fabes y Spinrad, 2006; Estell et al, 2002; Jiménez, 2003) y abandonan con mayor facilidad el ámbito escolar (Estévez, 2005; Garaigordobil, 2005b). Además, los jóvenes que se alejan del grupo de iguales por presentar ansiedad social también tienen problemas en su rendimiento académico. Así, la resistencia a participar en situaciones escolares, tales como realizar presentaciones orales, participar en debates y hacer preguntas en clase, puede contribuir al absentismo escolar, disminuyendo significativamente el rendimiento académico de los jóvenes (Amerigen, Manzini y Farvorden, 2003 ; Pastorelli et al., 2002).

En este marco académico, los padres son una figura importantísima para la consecución de las metas académicas en los jóvenes. La educación y el apoyo de los padres juega un papel relevante en el éxito académico (Adeyemo, 2005; González-Pienda, et al., 2002; González-Pienda., et al, 2003; Supple, et al., 2004). Un estudio reciente de Moilanen (2005), halló que la calidez de los padres y el control conductual esta asociado a la autorregulación y al rendimiento académico a largo plazo. Así pues, desde nuestro punto de vista los padres que tienen un estilo democrático de actuación ante los problemas escolares de sus hijos, y actúan en base a la firmeza, la comunicación y la colaboración, crean un mayor apoyo que contribuye a la constancia del estudiante en el estudio y a la superación de los momentos críticos.

Conforme ese joven se va convirtiendo en adulto sus metas y motivaciones académicas y laborales se van transformando. Así, a principios de la edad adulta el individuo comienza a buscar un trabajo que le va a proporcionar la estabilidad económica y una mayor inde- 
pendencia. En la edad adulta esta motivación es fundamental para sentirse competentes socialmente. Al conseguir un trabajo el adulto comienza a estabilizarse y adquirir su rol laboral dentro del grupo de trabajadores. Al igual que pasaba en el ámbito educativo, el adulto busca la aprobación y la aceptación en su trabajo. En el ámbito del trabajo, el apoyo de los compañeros es fundamental para conseguir una motivación laboral óptima y, por lo tanto, para conseguir un mayor rendimiento laboral. En este sentido, la Psicología del Trabajo y las Organizaciones ha estudiado la importancia de la cooperación para alcanzar un buen clima laboral entre los compañeros y lograr unos niveles óptimos en el rendimiento empresarial (Sánchez, 2007).

\section{Conclusiones}

Podemos concluir que, dentro del marco de las relaciones sociales, tanto los amigos como los padres juegan un papel clave en el bienestar emocional, físico, así como en el rendimiento académico y laboral. Ambas influencias terminan por ser complementarias en la vida de los jóvenes y los adultos. Además, dichas influencias evolucionan a lo largo de los años sin dejar de tener importancia.

De igual forma, el estilo educativo que ejercen los padres desde la juventud tiene una importancia especial en el desarrollo del estado emocional, relación social y rendimiento académico. Así, un estilo de educativo democrático reporta al joven una estabilidad emocional y un apoyo en las metas académicas, respecto a otros estilos educativos basados en la cohesión o en el permisivismo, que desembocan finalmente en conductas más desajustadas social y emocionalmente y en el rendimiento escolar.

Además, el grupo de amistades también juega un papel importantísimo en las relaciones sociales, el autoconcepto, la autoestima, las metas académicas y el rendimiento académico y laboral. En la juventud, los procesos de socialización comienzan a crearse mediante grupos de amigos con un motivo común de afiliación. El joven tenderá a seguir la tendencia del grupo entrando en juego aspectos como el liderazgo, los roles dentro del grupo, el miedo al rechazo, etc. Así, dependiendo del tipo de actitudes, conductas y metas que tenga el grupo de amistades respecto a la salud, el futuro académico, etc., la influencia de los iguales puede declinarse hacia un lado u otro de la balanza académica, física y/o emocional. Así, la influencia 
de los padres y los amigos van a tener un papel decisivo en el futuro académico y laboral del individuo.

Durante la edad adulta el individuo va pasando por una serie de etapas muy relacionadas con su motivación en la vida. El individuo irá teniendo motivaciones distintas, como conseguir un trabajo estable que le reporte dinero para obtener cosas que desea, una pareja y formar una familia. Las relaciones sociales van variando; se pasa de perseguir metas relacionadas con la aprobación de los iguales y la pertenencia a un grupo, a metas relacionadas con el aumento del estatus social y la competencia en el ámbito laboral. Si cabe, conforme aumenta la edad, el adulto se centra en metas más inmediatas, familiares y lúdicas, apareciendo un deseo de alejamiento en las relaciones sociales.

Respecto a la estabilidad emocional y la salud física, queremos destacar la importancia del estilo asertivo y prosocial, en el sentido de que los jóvenes más asertivos y prosociales tienen una mayor estabilidad emocional, deciden de forma adecuada respecto a su salud y tienen mejor rendimiento académico, respecto a los jóvenes que tienen un estilo basado en la agresividad o en la pasividad. Sin embargo, en la edad adulta esta estabilidad emocional se ve incrementada, aunque conforme pasan los años aumenta el riesgo de enfermedades vasculares $\mathrm{y}$, sobre todo, en aquellas personas con rasgos de personalidad tipo A. 


\section{Referencias}

Adeyemi, B.A. (2008). Efectos del aprendizaje cooperativo y las estrategias de resolución de problemas en el rendimiento de estudiantes del primer ciclo de secundaria en ciencias sociales. Electronic Journal of Research in Educational Psychology, 16, 6(3), 691708.

Adeyemo, D. A. (2005). La implicación de los padres, el interés en la escolarización y en el entorno escolar como factores determinantes de la auto-eficacia académica. Electronic Journal of Research in Educational Psychology, 5, 3(1), 163-180.

Alvarez D.M., Benavides J.L, Bueno, O., Cuadros V.P., Echeverri, D.R., Ibáñez L.G., Guzmán A.P.,Torres, M.P , Castaño J.J., Páez M.A. y Aristizábal J.F.(2006). Estudio comparativo del nivel de ansiedad, personalidad tipo a y factores de riesgo asociados a hipertensión arterial en pacientes hipertensos y no hipertensos. Archivos de medicina, $51-67$.

Amerigen, M.V., Manzini, C., y Farvorden, P. (2003).The impact of anxiety disorders on educational achievement. Journal of Anxiety Disorders, 17,561-571.

Argyle, M. y Lo, L. (1990). Happines and social skills. Personality and Individual Differences, $11,1255-1261$.

Battistich ,V., Schaps, E. y Wilson,N.(2004).Effects of an Elementary School Intervention on Students' "Connectedness" to School and Social Adjustment During Middle School. Journal of primary prevention, 24(3), 243-262.

Bergen, H.A., Martin, G., Roeger, L. y Allison, S. (2005). Perceived academic performance and alcohol, tobacco and marijuana use: Longitudinal relationships in young community adolescents. Addictive Behaviors, 30, 1563-1573.

Bijnen, F.C., Caspersen, D.J. y Mosterd, W.L. (1994). Physical inactivity as a risk factor for coronary heart disease: a WHO and International Society and Federation of Cardiology position statement. Bull World Health Organization, 72, 1-4.

Bijstra, J.O., Bosma, H.A. y Jackson, S. (1994). The relationship between social skills and psycho-social functioning en early adolescent. Personality and Individual Differences, $16,767-776$.

Bijstra, J.O., Jackson, S. y Bosma, H.A. (1995). Social skills and psycho-social functioning in early adolescence: A three-year follow-up. International Journal of Adolescent Medicine and Health, 8, 221-233. 
Blum, R.W. e Ireland, M. (2004). Reducing risk, increasing protective factors: Findings from the Caribbean Youth Health Survey. Journal of Adolescent Health, 35, 493-500.

Boliang, G., Yan, W., y Lei, Ch. (2005). Classroom effects on the relations between childrens social behaviors and school adjustment. Acta psychologica sinica, 37(2), 233-239.

Bracken, B. A. y Crain, R. M. (1994). Children's and adolescents' interpersonal relations: Do age, race, and gender define normalcy? Journal of Psychoeducational Assessment, 12, 14-32.

Brody, G.H., Chen, Y.F., Murry, V.M., Ge, X., Simons, R., Gibbons, F.X., Gerrard, M. y Cutrona, C.E. (2006). Perceived Discrimination and the Adjustment of African American Youths: A Five-Year Longitudinal Analysis with Contextual Moderation Effects. Child development, 7(5), 1170-1189.

Buhrmester, D. y Furman, W. (1992). The development of companionship and intimacy.Child Development, 58, 1101-1113.

Buhs, E.S., Ladd, G.W. y Herald, S.L. (2006). Peer exclusion and victimization: processes that mediate the relation between peer group rejection and children's classroom engagement and achievement ?. Journal of educational psychology, 98, 1-13.

Bulik, C.M., Beidel, D.C., Duchmann, E., Weltzin, T.E. y Kaye, W.H. (1991). An analysis of social anxiety in anorexic, bulimic, social phobic and control women. Journal of Psychopathology and Behavioral Assessment, 13, 199-211.

Bull World Health Organ (2000). Comparación transnacional de la prevalencia de los trastornos mentales y los factores con ellos correlacionados: estudio del ICPE. 78, 4, 413426.

Calvo, A.J., González, R. y Martorell, C. (2001). Variables relacionadas con la conducta prosocial en la infancia y adolescencia: Personalidad, autoconcepto y género. Infancia y Aprendizaje, 24(1), 95-111.

Clark, D.B. (1993, marzo). Assessment of social anxiety in adolescent alcohol abusers. Comunicación presentada en la Convención Anual de la Asociación Americana de Trastornos de Ansiedad. Charleston, USA.

Clark, D.B., Turner, S.M., Beidel, D.C., Donovan, J.E., Kirisci, L. y Jacob, R.G. (1994). Reliability and validity of the Social Phobia and Anxiety Inventory for Adolescents. Psychological Assessment, 6, 135-140.

Clark-Lempers, D.S., Lempers, J.D. y Ho, C. (1991). Early, middle, and late adolescents' perceptions of their relationships with significant others. Journal of Adolescent Research, 6, 296-315. 
Chen, K. (2006). Social Skills Intervention for Students with Emotional/Behavioral Disorders: A Literature Review from the American Perspective. Educational Research and Reviews, 1(3), 143-149.

Chen, X., Chang, L. y He, Y. (2003). The peer group as a context: mediating and moderating effects on relations between achievement and social functioning in Chinese children. Child development, 74(3), $710-727$.

Dana L. H., Scott J. S. y Sunita, B. (2006). The company you keep: adolescent mobility and peer behavior. Sociological inquiry, 76(3), 397-426.

Del Barrio, C., Martín, E., Almeida, A., y Barrios, A. (2003). Peer maltreatment and other concepts related to school aggression and their psychological study. Infancia y Aprendizaje, 26(1), 9-24.

De Micheli, D. y Formigoni, M.L. (2004). Drug use by Brazilian students: Associations with family, psychosocial, health, demographic and behavioral characteristics. Addiction, 99, 570-578.

Eisenberg, N. (1990). Prosocial development in early and mid-adolesence. En R. Montemayor, G.R. Adams y T. P. Gullotta (Eds.), From childhood to adolescence: a transitional period. Beverly Hills: Sage.

Eisenberg, N., Fabes, R. A. y Spinrad, T. L. (2006). Prosocial development. En W. Damon y N. Eisenberg (Eds.), Handbook of child psychology, Vol. 3: Social, emotional and personality development (pp. 646-718). New York: John Wiley \& Sons.

Eisenberg, N., Carlo, G., Murphy, B. y Van Court, P. (1995). Prosocial development in late adolescence: A longitudinal study. Child development, 66, 1179-1197.

Eisenberg. N., Miller.P.A., Shell.R., McNalley.S. y Shea.C. (1991). Prosocial development in adolescence: A longitudinal study. Developmental psychology, 27(5), 849-857.

Eisenberg, N., Zhou, Q. y Koller, S. (2001). Brazilian adolescents' prosocial moral judgment and behavior: Relations to sympathy, perspective taking, gender-role orientation, and demographic characteristics. Child development, 73, 518-534.

Erikson, E.H. (1985). El ciclo vital completo. Mexico: Paidos.

Estell, D., Farmer, Th. W., Cairns, R. B y Cairns, B. D.(2002) Social relations and academic achievement in inner-city early elementary classrooms. International journal of behavioral development, 26(6), $518-528$.

Estévez E. (2005). Violencia, victimización y rechazo escolar en la adolescencia. Universitat de Valencia. Departamento de Psicología Social. Servei De Publicacions. 
Fernández, F., Jiménez, S., Badía, A., Jaurrieta, N., Solano, R., y Vallejo, J. (2003).Ansiedad y fobia social en anorexia nerviosa: Un estudio de casos y controles. Psicología Conductual, 11,517-525.

Flores, M. F. y Díaz, R. D. (1995). Desarrollo y validación de una Escala Multidimensional de Asertividad para Estudiantes. Revista Mexicana de Psicología, 12, 133-144.

Furman, W. y Buhrmester, D. (1992). Age and sex differences in perceptions of networks of personal relationships. Child Development, 63, 103-115.

Galambos, N.L. y Almeida, D.N. (1992). Does parent-adolescent conflict increase in early adolescence? Journal of Marriage and the Family, 54, 737-747.

Garaigordobil, M. (2005). Diseño y evaluación de un programa de intervención socioemocional para promover la conducta prosocial y prevenir la violencia. Madrid: Centro de Publicaciones del Ministerio de Educación y Ciencia. Colección Investigación No 160 (Primer Premio Nacional de Investigación Educativa 2003).

Garaigordobil, M. (2005b). Conducta antisocial durante la adolescencia: correlatos socioemocionales, predictores y diferencias de género. Psicología conductual, 13(2), 197221.

Garaigordobil, M., Cruz, M.S. y Pérez, J.I. (2003). Análisis correlacional y predictivo del autoconcepto con otros factores conductuales, cognitivos y emocionales de la personalidad durante la adolescencia. Estudios de psicología, 24, 113-134.

Gilman, R., y Huebner, E. S. (2006). Characteristics of adolescents who report high life satisfaction. Journal of Youth and Adolescence, 35, 311-319.

González-Pienda, J.A., Núñez, J.C., Alvarez, L. y González-Pumariega, S. (2002).Inducción parental a la autorregulación, autoconcepto y rendimiento académico. Psicothema, $14(4), 853-860$.

González-Pienda.J.A., Núñez .J.E., Álvarez.L., Roces, C., González-Pumariega.S., González. P., Muñiz.R., Valle. A., Cabanach.R.G., Rodríguez.S. y Bernardo.A. (2003). Adaptabilidad y cohesión familiar, implicación parental en conductas autorregulatorias, autoconcepto del estudiante y rendimiento académico. Psicothema, 15(3), 471-477.

Griffin, K.W., Botvin, G.J., Epstein, J.A., Doyle, M.M. y Diaz, T. (2000). Psychosocial and behavioral factors in early adolescence as predictors of heavy drinking among high school seniors. Journal of Studies on Alcohol, 61, 603-606.

Harrier, L.K., Lambert, P.L. y Ramos, V. (2001). Indicators of adolescent drug users in a clinical population. Journal of Child and Adolescent Substance Abuse, 10, 71-87. 
Hoffman, L., Paris, S. y Hall, E.(1996). Psicología del Desarrollo Hoy. Madrid : McGrawHill.

Huberman, A. M. (1974). La educación de adultos desde la perspective de su ciclo vital. En International Review of Education, Vol. XX.

Huebner, E. S., Suldo, S., y Gilman, R. (2006). Life satisfaction among children and adolescents. In G. Bear and K. Minke (Eds.), Children's Needs (3rd ed.) (pp.357-368). Washington, DC: National Association of School Psychologists.

Inderbitzen, H. M., Walters K. S. y Bukowski, A. L. (1997). The role of social anxiety in adolescent peer relations: Differences among sociometric status groups and rejected subgroups. Journal of Clinical Child Psychology, 26, 338-348.

Inglés, C.J. (2009). Enseñanza de Habilidades Interpersonales en la adolescencia. Programa PEHIA. $3^{\text {a }}$ Ed. Madrid: Pirámide.

Inglés, C.J., Benavides, G., Redondo, J., García-Fernández, J.M., Ruiz-Esteban C., Estévez C. y Huescar, E. (2009). Conducta prosocial y rendimiento académico en estudiantes españoles de Educación Secundaria Obligatoria. Anales psicología. 25, 93-101.

Inglés, C.J., Delgado, B., Baustista, R., Torregrosa, M.S., Espada, J.P., García-Fernández, J.M. et al. (2007). Factores psicosociales relacionados con el consumo de alcohol y tabaco en adolescentes españoles. Internacional Journal of Clinical and Health Psycho$\log y, 21,11-22$.

Inglés, C.J., Delgado, B., García-Fernández, J. M., Ruiz-Esteban, C. y Díaz-Herrero, A. (en prensa). Sociometric types and social interaction styles in a sample of Spanish adolescents. The Spanish Journal of Psychology.

Inglés, C.J., Hidalgo, M.D., Méndez, F.X. e Inderbitzen, H.M. (2003). The Teenage Inventory of Social Skills: Reliability and validity of the Spanish translation. Journal of Adolescence, 26, 505-510.

Inglés, C.J., Hidalgo, M.D. y Méndez, F.X. (2005). Interpersonal difficulties in adolescence: A new self-report measure. European Journal of Psychological Assessment, 21, 11-22. Inglés, C.J., Méndez, F.X., Hidalgo, M.D., y Spence, S.H. (2003).The List of Social Situation Problems: Reliability and validity in an adolescent Spanish-speaking sample. Journal of Psychopathology and Behavioral Assessment, 25, 65-74.

Inglés, C. J., Ruiz, C., García, J. M., Benavides, G., Estévez, C., Martínez, F., Torregrosa, M. S. y Pastor, Y. (2005). Tasas de popularidad, rechazo y olvido en estudiantes prosociales de E.S.O.. En J. A. Del Barrio, M. I. Fajardo, F. Castro, A. Díaz e I. Ruiz (Eds.), 
Nuevos Contextos Psicológicos y Sociales en Educación (pp. 323-335). Extremadura: PSICOEX.

Jessor, R. (1998). New perspectivas on adolescent risk behavior. New York: Cambridge University Press.

Jiménez, M. (2003). Características emocionales y comportamentales de los grupos sociométricos desde una perspectiva múltiple. Psicología Conductual, 11(1), 41- 60.

Katainen, S., Räikkönen, K \& Keltikangas-Järvinen, L. (1999). Adolescent temperament, perceived social support, and depressive tendencies as predictors of depressive tendencies in young adulthood. European Journal of Personality, 13, 183-207.

Kimmel, D.C. y Weiner, I.B. (1998). La adolescencia: Una transición del desarrollo. Barcelona: Ariel.

Kollins, S.H., McClernon, F.J. y Fuemmeler, B.F. (2005). Association between smoking and attention-deficit/hyperactivity disorder symptoms in a population-based sample of young adults. Archives of General Psychiatry, 62, 1142-1147.

Kupersmidt, J., Coie, J.D. y Dodge, K.A. (1990). The role of poor peer relationships in the development of disorder. In S.R. Asher y J.D. Coie (Eds.). Peer rejection in childhood (pp. 274-305). Nueva York: Cambridge University Press.

Ladd, R.M. (1990). Having friends, keeping friends, making friends, and being liked by peers in the classroom: Predictors of children's early school adjustment? Child development, $61,1081-1100$.

Ladd, R.M. y Kochenderfer, B.J. (1996). Linkages between friendship and adjustment during early school transitions. En W.M.Bukowski, A.F.Newcomb, y W.W.Hartup (eds.). The company they keep ( pp. 322-345).New York: Cambridge University Press.

La Greca, A.M., Prinstein, M.J. y Fetter, M.D. (2001). Adolescent peer crowd affiliation: Linkages with health-risk behaviors and close friendships. Journal of Pediatric Psychology, 26, 131-143.

La Greca, A. M. y López, N. (1998). Social anxiety among adolescents: Linkages with peer relations and friendships. Journal of Abnormal Child Psychology, 26, 83-94.

Laursen, B., Coy, K.C. y Collins, W.A. (1998). Reconsidering changes in parent-child conflict across adolescence: A meta-analysis. Child Development, 69, 817-832.

Lee, C.D., Folsom, A.R. y Blair, S.N.(2003). Physical activity and stroke risk: a metaanalysis. Stroke. 35, 79-80. 
Lempers, J.D. y Clark-Lempers, D.S. (1992). Young, middle, and late adolescents' comparisons of the functional importance of five significant relationships. Journal of Youth and Adolescence, 21, 53-96.

Lin,-Shan. (2001). The influence of family connection, regulation, and psychological control on Chinese adolescent development. Humanities and social sciences, 62(6-A), 2249.

Lynch, M. y Cicchetti, D. (1997). Children's relationships with adults and peers: An examination of elementary and junior high school students. Journal of School Psychology, 35, 81-89.

Markiewicz, D., Doyle, A. B. y Brendgen, M. (2001). The quality of adolescents' friendships: Associations with mothers' interpersonal relationships, attachments to parents and friends, and prosocial behaviour. Journal of Adolescence, 24, 429-445

Martínez, A. E. (2009). Autoconcepto, motivación académica y estrategias de aprendizaje en estudiantes prosociales y no prosociales de educación secundaria obligatoria. Tesis Doctoral. Universidad Miguel Hernández. Elche. España.

Martínez-González, J.M., Robles-Lozano, L. y Trujillo, H.M. (2003). Diferencias sociodemográficas y protección ante el consumo de drogas legales. International Journal of Clinical and Health Psychology, 3, 461-475.

Mayseless, O., Wiseman, H. y Hai, I. (1998). Adolescents' relationships with father, mother, and same-gender friend. Journal of Adolescent Research, 13, 101-123.

Méndez, F ,X ., Inglés, C. J ., e Hidalgo, M. D .(2002). Estrés en las relaciones interpersonales: un estudio descriptivo en la adolescencia. Ansiedad y estrés, 8, 1, 25-36.

Mestre M.V., Tur, A.M.,Samper, P.,Nácher M.J. y Cortés M.T. (2007). Estilos de crianza en la adolescencia y su relación con el comportamiento prosocial. Revista latinoamericana de psicología, 39, 2.

Moilanen,-Kristin-Lea. (2005). Parenting and self-regulation in adolescence: Associations with adolescent behavior. The sciences and engineering, 66(5-B), 2858.

O'Callaghan, F. y Doyle, J. (2001). What is the role of impression management in adolescent cigarette smoking? Journal of Substance Abuse, 13, 459-470.

Ollendick, T.H., Weist, M.D., Borden, C. y Greene, R.W. (1992). Sociometric status and academic, behavioral, and psychological adjusment: A five-year longitudinal study. Journal of Consulting and Clinical Psychology, 60, 80-87.

Paschall, M.J., Flewelling, R.L. y Russell, T. (2004). Why is work intensity associated with heavy alcohol use among adolescents? Journal of Adolescent Health, 34, 79-87. 
Pastorelli,C., Gerbino,M., Vecchio,G.M., Steca,P., Picconi,L. y Paciello,M .(2002). L'insuccesso scolastico: Fattori di rischio e di protezione nel corso della preadolescenza / School under-achievement: Risk and protective factors during adolescence . Eta Evolutiva, 71(1), 84-91.

Rabe-Jablonska, J., Dietrich-Muszalska, A. y Gmitrowicz, A. (2004). The prevalence of social phobia in a representative group of adolescents from Lodz. Archives of Psychiatry and Psychotherapy, 6, 15-22.

Redondo, J. (2007).Conducta prosocial, atribuciones causales y rendimiento académico en E.S.O. Tesis Doctoral.Universidad Miguel Hernández. Elche. España.

Rice, P. (1997). Desarrollo humano. Estudio del ciclo vital. México: Prentice Hall.

Rice, K.G y Mulkeen, P.(1995). Relationships with parents and peers: A longitudinal study of adolescent intimacy. Journal of Adolescent Research, 10, 338-357.

Rodrigo, M.J., Máiquez, M.L., García .M., Mendoza. R., Rubio.A., Martinez.A y Martín J.C. (2004). Relaciones padres-hijos y estilos de vida en la adolescencia. Psicothema, $16(2), 203-210$.

Sanchez, M.A. (2007). Clima laboral y estrategia de comunicación. Comunicación organizacional, 1,1, http: web.upaep.mx/revistaeyc/organizacional.htm

Schroeder, K.E., Masahiko, k., Davison D., Pesek, C., Bradley F., Narkiewicz,K, Pesek, C., Phillips, B; Davison, D. y Somers, V.K. (2000) Personality type and neural circulatory. Hypertension, 36, 820- 833.

Siverio, M, A. y García, M, D. (2007). Autopercepción de adaptación y tristeza en la adolescencia: La influencia del género. Anales de psicología, 23, 1, 41-48

Sletta, O., Valas, H. y Skaalvik, E. (1996). Peer relations, loneliness, and self-perceptions in school-aged children. British Journal of Educational Psychology, 66, 431-445.

Smetana, J.G. (1989). Adolescents' and parents' reasoning about actual family conflict. Child Development, 60, 1052-1067.

Smetana, J.G. (1993). Conceptions of parental authority in divorced and married mothers and their adolescents. Journal of Research on Adolescence, 3, 19-39.

Smetana, J.G., Braeges, J.L. y Yau, J. (1991). Doing what you say and saying what you do: Reasoning about adolescent-parent conflict in interviews and interactions. Journal of Adolescence Research, 6, 276-295.

Smith, E. P., Walker, K., Fields, L., Brookings, C.C. y Seay, R.C. (1999). Ethnic identity and its relationship to self-esteem, perceived efficacy and prosocial attitudes in early adolescence. Journal of Adolescence, 22(6), 867-880. 
Sonntag, H., Wittchen, H.U., Höfler, M., Kessler, R.C. y Stein, M.B. (2000). Are social fears and DSM-IV social anxiety disorder associated with smoking and nicotine dependence in adolescents and young adults? European Psychiatry, 15, 67-74.

Storch, E, A., Masia-Warner, C., Crisp, H. y Klein, R, G. (2005). Peer victimization and social anxiety in adolescence: a prospective study. Aggressive Behavior, 31(5), 437 - 452.

Supplee L H., Shaw. D S., Hailstones. K. y Hartman K. (2004). Family and child influences on early academic and emotion regulatory behaviors. Journal of School Psychology. $42,221-242$

Sussman, S., Unger, J.B. y Dent, C.W. (2004). Peer group self-identification among alternative high school youth: A predictor of their psychosocial functioning five years later. International Journal of Clinical and Health Psychology, 4, 9-25.

Tennant, S.M. (2005). Peer orientation towards school, students' achievement goals, and adolescents' achievement-related behavior. The sciences and engineering, 66(3-B), 1789.

Trianes. M.V., Blanca. M.J., Muñoz. A., García. B., Cardelle-Elawar.M. e Infante.L. (2002). Relaciones entre evaluadores de la competencia social en preadolescentes: Profesores, iguales y autoinformes. Anales de Psicología, 18(2), 197-214.

Tur, A., Mestre, M. V. y del Barrio, V. (2004). Factores moduladores de conducta agresiva y prosocial. El efecto de los hábitos de crianza en la conducta del adolescente. Ansiedad y Estrés, 1,78-88.

Upadhyaya, H.P., Brady, K.T., Wharton, M. y Liao, J. (2003). Psychiatric disorders and cigarette smoking among child and adolescent psychiatry inpatients. American Journal on Addictions, 12, 144-152.

Vandell, D.L., y Mueller, E.C. (1980). Peer play and friendship during the first two years. En H.C.Foot, A.J.Chapman, y J.R.Smith (Eds.), Friendship and social relations in children (pp.181-208).Nueva York: Wiley.

Velásquez, E., Martínez, M.L. y Cumsille, P. (2004). Expectativas de autoeficacia y actitud prosocial asociadas a participación ciudadana en jóvenes. Revista de la Escuela de Psicología, 13(2), 85-98.

Volling, B.L., Youngblade L.M. y Belsky, J. (1997). Young children's social relationships with siblings and friends. American Journal of Orthopsychiatry, 61, 102-111.

Walters, K. S. e Inderbitzen, H. M. (1998). Social anxiety and peer relations among adolescents: Testing a psychobiological model. Journal of Anxiety Disorders, 12, 183-198.

Weiller, E., Bisserbe, J.C., Boyer, P., Lepine, P. y Lecrubier, Y. (1996). Social phobia in general health care. British Journal of Psychiatry, 168, 169-174. 
Wentzel, K.R., Barry, C.M y Caldwell, K.A. (2004). Friendships in Middle School: Influences on Motivation and School Adjustment. Journal of Educational Psychology. 96(2), 195-203.

Wills, T.A. y Resko, J.A. (2004). Social support and behavior toward others: Some paradoxes and some directions.En A.G.Miller (Ed.), The social psychology of good and evil (pp.416-443).New York, US: Guilford Press.

Wills T, A., Resko J, A., Ainette M, G. y Mendoza D. (2004) .Role of parent support and peer support in adolescent substance use: a test of mediated effects. Psychology Addiction Behavioral. 18(2):122-34.

Wittchen, H.U., Stein, M.B. y Kessler, R.C. (1999). Social fears and social phobia in a community sample of adolescents and young adults: Prevalence, risk factors and comorbidity. Psychological Medicine, 29, 309-323.

Wu, L.T., Schlenger, W.E. y Galvin, D.M. (2003). The relationship between employment and substance use among students aged 12 to 17. Journal of Adolescent Health, 32, 5-15.

Zimmermann, P., Wittchen, H.U., Höfler, M., Pfister, H., Kessler, R.C. y Lieb, R. (2003). Primary anxiety disorders and the development of subsequent alcohol use disorders: A 4-year community study of adolescents and young adults. Psychological Medicine, 33, 1211- 1222. 